\title{
OPERATIONAL EXPERIENCE WITH RESONANTLY EXTRACTED BEAMS*
}

\author{
K. D. Jacobs, F. Casagrande, M. Farkhondeh, B. G. McAllister, L. W. O’Brien, \\ C. Tschalaer, E. Tsentalovich, F. Wang, and G. T. Zwart \\ MIT-Bates Linear Accelerator Center, Middleton, MA 01949
}

\begin{abstract}
The MIT-Bates Linear Accelerator has completed a major nuclear physics experiment using high duty factor electron beam resonantly extracted from the South Hall Ring. Average currents up to $8 \mu \mathrm{A}$ at energies from 569 to $950 \mathrm{MeV}$ were delivered to the new Out Of Plane Spectrometer System, with typical duty factors of $60 \%$. Throughput efficiencies, measured as the ratio of beam current injected into the ring to that delivered to experiment, were $80-90 \%$. The extraction process was repeated at a $600 \mathrm{~Hz}$ repetition rate, giving storage times much shorter than the beam damping time. Thus, beam dynamics were exclusively in a transient state. Details of the operational experience, including important aspects of beam set-up, on-line monitoring, and maintenance, are presented.
\end{abstract}

\section{INTRODUCTION}

The MIT-Bates Linear Accelerator Center comprises a pulsed S-band electron linac and recirculator capable of unloaded beam energies up to $1.1 \mathrm{GeV}$, for basic research in nuclear physics. Typical duty factors of the beam from the linac are on the order of $1 \%$. With the current interest in coincidence experiments, it is desirable to increase the duty factor of the beam to experiment, while maintaining the same average current. To that end, we have constructed the South Hall Ring (SHR) to function as a pulse stretcher, using resonant extraction to convert the pulsed beam from the linac/recirculator to quasi-CW beam. In late calendar 2000 and early 2001, the first two physics experiments using extracted beam from the SHR were completed. These experiments used beam at six different energies, with average currents typically around $7 \mu \mathrm{A}$ and duty factors of $50 \%$.

\section{DESCRIPTION}

The pulse-stretching process is accomplished using half-integer extraction. The linac and recirculator are operated in "head-to-tail" mode, wherein the beam pulse width is carefully adjusted (to $\sim 10 \mathrm{~ns}$ precision) so that the leading edge of the pulse on its second pass through the linac follows immediately behind the trailing edge of the pulse on its first pass through the linac. This gives a beam pulse width of $1.3 \mu$ s at injection into the ring,

\footnotetext{
* Work supported by the U.S. Department of Energy cooperative agreement DE-FC02-94ER40818.A000
}

which is twice the ring circumference. Thus, we use twoturn injection into the ring.

At injection, the horizontal tune of the ring is set to 7.460. A ferrite core quadrupole is then ramped to drive the tune towards the half-integer resonance (7.500). Octupoles located in a non-dispersive straight section divide the horizontal phase space into a central stable region, and an outer unstable region. The beam is injected into this phase space such that it is just inside the separatrix dividing the stable and unstable regions. As the tune is driven toward the half integer, the separatrix moves across the beam, gradually causing the electron motion to become unstable in the $x$ direction. As an unstable electron's betatron amplitude grows, it will cross the $100 \mu \mathrm{m}$ thick extraction electrostatic septum, and be extracted from the ring and delivered to the experiment. The time profile of the ramped quadrupole is adjusted so that after $1.6 \mathrm{~ms}$ the ring is empty. At that time another pulse from the linac is injected into the ring, and the cycle repeats again. Thus, the repetition rate for the extraction process is $600 \mathrm{~Hz}$.

\subsection{Considerations}

Delivering beam as described above is a delicate process. There are tight tolerances on the position, angle, centroid energy, and energy spread of the beam injected into the ring. Deviations from nominal values can result in excessive beam loss, poor extraction efficiency, low duty factor, unacceptably high prompt extraction (too much current coming out in the first tens of microseconds after injection), or high backgrounds for the experiment.

In addition to the tight tolerances, the situation is complicated by the fact that time scales involved are too short for the beam to reach equilibrium. This occurs in two places: In the linac, where the beam pulse length is comparable to the RF filling time, and in the ring, where the extraction period is much less than the beam damping time.

In the linac, the RF filling time is $1.3 \mu$ s. Thus, the first microsecond or so of the beam pulse is at a higher energy, and must be dumped somewhere. This is typically done on energy defining slits in a dispersive region. When running with head-to-tail recirculation though, the desired energy analyzed pulse length is comparable to the beam loading time, resulting in high average currents being deposited on the slits.

In the ring, damping times are on the order of $100 \mathrm{~ms}$ at the highest operational energies, and substantially longer 
at lower energies. The $1.6 \mathrm{~ms}$ extraction period is much shorter than this, meaning that betatron and synchrotron oscillations resulting from injection off the synchronous coordinates do not have time to damp out.

\subsection{Solutions}

The extensive running during the past year and a half has given us considerable experience in dealing with these issues. The first step is to ensure that the various beamlines are properly set up. In particular, in the ring there are multiple optical conditions which must be simultaneously met: The horizontal tune must be 7.46, $\beta_{\mathrm{x}}$ must be large $(\sim 50 \mathrm{~m})$ at the extraction septum and $\sim 25 \mathrm{~m}$ at the injection septum, with the proper phase advances between the septa and the ocutpoles used for extraction, and so on. Setting the ring magnets to values based on magnetic measurements did not produce the necessary optical functions. To solve this, beam-based optics measurements (LOCO: Linear Optics from Closed Orbit [1]) were successfully employed to properly set the ring quadrupoles to achieve the desired optics.

Once a proper optical set-up had been established at one energy, scaling to different energies was accomplished using an on-line procedure, directly interfaced with the accelerator control system (EPICS). This allowed us to efficiently and reproducibly change energies [2].

One of the key elements in the success of this run was the extensive on-line monitoring of a wide variety of beam parameters, once the beam was properly established. These included:

- Continuous monitoring of the extraction efficiency. A toroid measured the current injected into the ring, and a Faraday Cup located at the end of the beamline measured the current to the experiment. Both these signals were digitized and displayed via EPICS. In addition, the experimenter's gate defining usable beam was monitored to give a measure of the beam being used by the experiment. (It is necessary to exclude from the gate the first $100 \mu$ s or so of each extraction cycle, to avoid high peak currents due to some prompt extracted beam.)

- Duty factor monitoring. One method of doing this is to compare the singles and coincidence rates in two detectors. This provides a numeric (digital) measure. A more useful monitor, though less quantitative, was to observe on an oscilloscope the profile of the extracted current as a function of time, over the $1.6 \mathrm{~ms}$ extraction cycle. This was most readily accomplished using the analog signal from a photoemission monitor (PEM) making use of the synchrotron radiation in an extraction line dipole magnet [3]. Beam position monitors (BPMs) in the extraction line, capable of making measurements down to $1 \mu \mathrm{A}$ peak current, were also able to monitor the beam current and produce an analog signal similar to that from the PEM. However the BPM makes use of the radio frequency (RF) structure of the beam. Thus, if some of the beam was not caught in the ring RF bucket, (which did happen from time to time), that beam would go undetected by the BPM.

- Several monitors of experimental background levels were available, and showed when the quality of the beam was deteriorating. Also, keeping track of known sources of background, such as injected beam striking the injection electrostatic septum, allowed for fast determination of some sources of background.

- Monitoring the beam current stored in the ring, on widely different time scales, was important. On short time scales $(\sim 10 \mu \mathrm{s})$, a toroid in the ring showed the current injected into the ring, and the current for the first ten or so turns. Problems with the trajectory, energy, or energy spread of the injected beam were readily identifiable there. But it was also useful to monitor the current circulating in the ring over an entire extraction cycle $(1.6 \mathrm{~ms})$. For this, a DC Current Transformer was used. Each analog current signal was displayed on its own oscilloscope, along with other signals of similar time scales.

- Analog signals from BPMs in dispersive regions were used to observe the time structure of the beam pulse in the linac, recirculator, and beam switchyard. This was used to verify the effectiveness of the energy feedback systems (to remove $60 \mathrm{~Hz}$ energy jitter) [4], and the staggered timing system to deal with the linac beam loading transient (discussed below).

- Many of the signals described here were digitized and archived under EPICS. This allowed for analysis of long term trends (over hours or days), searches for correlations between various signals (both those described here and many others being monitored), and other useful diagnostics.

To deal with the beam loading transients in the linac, a new system for timing the linac RF was installed. The idea of this "staggered timing" system was to turn on the $\mathrm{RF}$ in selected sections of the linac at a time comparable to that of beam arrival at that section [5]. The electrons at the leading edge of the pulse, which would normally be at higher energy because the linac was not yet loaded down, have an energy closer to the electrons later in the pulse because the linac RF is still filling the accelerator. By carefully timing the RF turn-on in three or four sections of the linac, the $10 \%$ beam loading effect was reduced to less than $0.5 \%$.

\section{RESULTS}

All of the above monitors were routinely used in delivering the resonantly extracted beam to experiments, at six energies ranging from 569 to $950 \mathrm{MeV}$. Indeed, the majority of them were used continuously. Typical beam currents within the experimental gate were $7 \mu \mathrm{A}$, at roughly $50 \%$ duty factor. Figure 1 shows the extracted 
beam current over one extraction cycle, and the current in

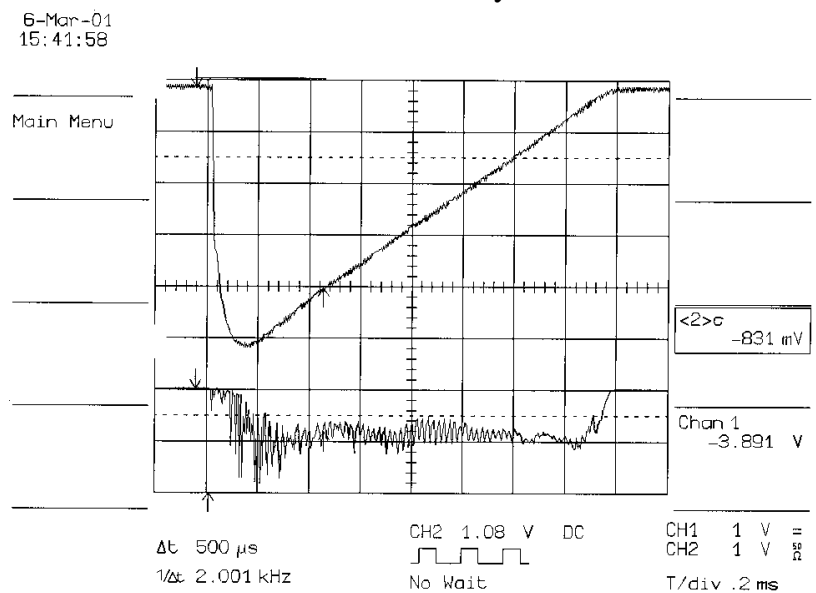

Figure 1: Stored beam in the SHR and extracted beam delivered to the OOPS experiment, during one extraction cycle. The horizontal scale is $0.2 \mathrm{~ms} / \mathrm{div}$. (top) Beam stored in the ring. Zero current is near the top of the figure, and current increases downward at $5 \mathrm{~mA} / \mathrm{div}$. The nearly straight line decrease in the current demonstrates how uniformly electrons were being extracted from the ring. (bottom) Current in the extraction line, as measured by the Photo Emission Monitor. Increasing current is down in the figure.

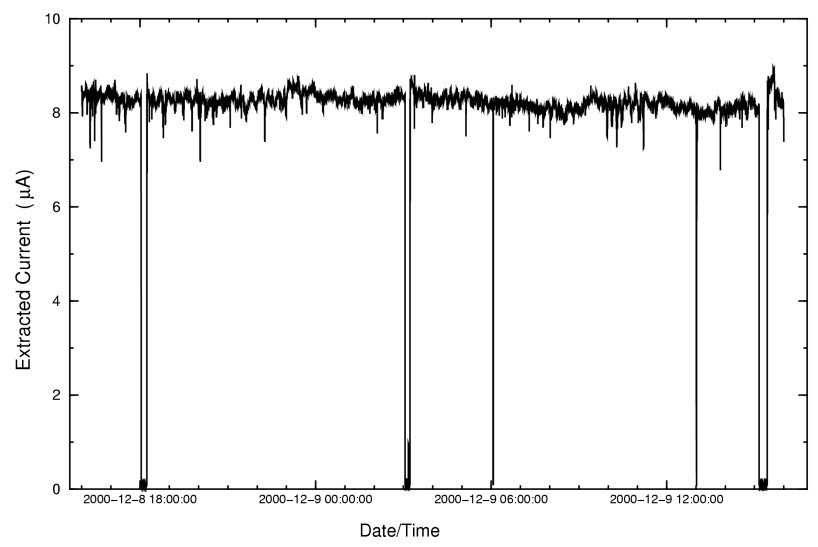

Figure 2: Extracted beam current measured by a Faraday Cup, at the end of the beam time. The time span is 24 hours. During the periods where the current goes to zero, the beam was intentionally turned off. the ring throughout the cycle. This demonstrates the flat extracted beam profile, without significant prompt extraction, which could be attained under good operating conditions. Beam delivered to experiment over a time scale of approximately one day is shown in Fig. 2. By paying close attention to the important beam parameters, it was possible to achieve highly efficient accelerator operation.

\section{SUMMARY}

Delivery of high energy resonantly extracted beam at MIT-Bates uses the facility at its maximum: It uses both the linac and recirculator, operated in head-to-tail mode, and the South Hall Ring in pulse-stretcher mode. Beam time scales are shorter than or comparable to the relevant equilibrium times. Tolerances on beam position, angle, energy, and energy spread, are tight. Nonetheless, by carefully monitoring selected beam parameters, it was possible to complete two major medium-energy nuclear physics experiments. This marks the first time the SHR has been used for physics experiments.

\section{REFERENCES}

[1] Safranek, J. A, "Beam-based Accelerator Modeling and Control", in Proc. of the 2000 Beam Instrumentation Workshop, edited by K. D. Jacobs and R. Coles Sibley III, 2000, pp. 23-35

[2] Wang, F., Jacobs, K. D., Bisson, E., Geng, X., Laan, J. B. van der, and Yang, B., "Web Browser Based Applications for the MIT-Bates Accelerator", these proceedings.

[3] Euteneuer, H., et al., "Beam Monitors at the Mainz Microtron", in Proc. of the 1992 Linear Accelerator Conf., AECL-10728, pp. 356-358.

[4] Barkhuff, D. H., et al., Nucl. Instr. Methods Phys. Res. A 450, 187 (2000).

[5] Kroes, F. B., Sluyk, T. G. B. W., and Heutenik, B., "Performance and Modifications of the MEA R.F. Drive System", in Proc. of the 1989 Particle Accelerator Conf., edited by F. Bennett and J. Kopta, 1989, pp. 1719-1721. 